COMMUNICATIONS IN

ANALYSIS AND GEOMETRY

Volume 13, Number 2, 363-378, 2005

\title{
On the Rectifiability of the Free Boundary of the One Phase Stefan Problem
}

\author{
Marianne K. Korten
}

\begin{abstract}
In one space dimension and for a given function $u_{I}(x) \in C_{0}^{\infty}$, (say such that $u_{I}(x)>1$ in some interval) the equation $u_{t}=\Delta(u-1)_{+}$ can be thought of as describing the energy per unit volume in a Stefan-type problem, where the latent heat of the phase change is given by $\left(1-u_{I}(x)\right)_{+}$. Given a solution in the sense of distributions $0 \leq u \in L_{\mathrm{loc}}^{1}\left(\mathbb{R}^{n} \times(0, T)\right)$ of this equation, $(u-1)_{+}$is a subsolution to the heat equation. The "loss" with respect to a caloric function is accounted for by a Radon measure $\lambda$ supported on the free boundary $\mathcal{F}=\partial\left\{(x, t):(u(x, t)-1)_{+}>0\right\}$. We prove that this measure is $n$ rectifiable, i. e., $\mathcal{F}$ is $\lambda$-essentially the union of images of imbedded $C^{1}$ manifolds of dimension $n$ in $\mathbb{R}^{n} \times(0, T)$, under a weak assumption on the spatial gradient of $(u-1)_{+}$.
\end{abstract}

\section{Introduction.}

In one space dimension, and for given initial datum $u_{I}(x) \in C_{0}^{\infty}$, (say, such that $u_{I}(x)>1$ in some interval) the equation

$$
u_{t}=\Delta(u-1)_{+}
$$

can be thought of as describing the energy per unit volume in a Stefantype problem, where the latent heat of the phase change is given by (1$\left.u_{I}(x)\right)_{+}$. Note that discontinuous solutions should be expected for (1.1) (see [BouKMar]).

In previous papers $([\mathrm{AnK}],[\mathrm{K}])$ a-priori regularity of non-negative solutions (in the sense of distributions) $u \in L_{\text {loc }}^{1}\left(\mathbb{R}^{n} \times(0, T)\right)$ of (1.1) was found: Specifically it was shown that $u, \nabla_{x}(u-1)_{+}$, and $\frac{\partial}{\partial t}(u-1)_{+} \in$ $L_{\text {loc }}^{2}\left(\mathbb{R}^{n} \times(0, T)\right)$. Continuity of $(u-1)_{+}$in $\left(\mathbb{R}^{n} \times(0, T)\right)$ then follows from DiBenedetto's result [Di]. It was aso shown that $(u-1)_{+}$is a (weak)

\footnotetext{
${ }^{1}$ AMS subject classifications. 35K65, 35B65, 31A20, 35A30, 49Q15.
} 
subsolution to the heat equation. From the weak Harnack inequality

$$
\int_{\mathbb{R}^{n}} u(x, t) \exp \left(-c|x|^{2}\right) d x \leq M(u, n, T),
$$

for some $c=c(T) \in \mathbb{R}_{+}$and $0<t<T / 2$ (see [AnK]), existence of a unique initial trace $0 \leq \mu(x)$ follows, a Radon measure satisfying the growth condition at infinity

$$
\int_{\mathbb{R}^{n}} \exp \left(-c|x|^{2}\right) d \mu(x)<\infty
$$

Conversely, each such measure gives rise to a solution of (1.1), which is unique (see $[\mathrm{K}]$ ). In $[\mathrm{K} 1]$ we prove that for all $\beta, k>0$ and $\mathcal{L}^{n}$ a. e. $x_{0} \in \mathbb{R}^{n}$, there exists

$$
\lim _{(x, t) \in \Gamma_{\beta}^{k}\left(x_{0}\right),(x, t) \rightarrow x_{0}}(u(x, t)-1)_{+}=\left(f\left(x_{0}\right)-1\right)_{+},
$$

where $\mathcal{L}^{n}$ denotes $n$-dimensional Lebesgue measure, $f=\partial \mu / \partial \mathcal{L}^{n}$ is the Radon - Nikodym derivative of the initial trace with respect to the $n$-dimesional Lebesgue measure, and $\Gamma_{\beta}^{k}\left(x_{0}\right)=\left\{(x, t):\left|x-x_{0}\right|<\beta \sqrt{t}, 0<\right.$ $t<k\}$ are parabolic "nontangential" approach regions.

In this paper we study the structure of the free boundary of the one phase Stefan problem formulated in enthalpic variables (1.1): $u$ stands for the energy (enthalpy), $(u-1)_{+}$for the temperature, and we allow for a variable latent heat at the interphase. The set $\{(x, t): 0 \leq u(x, t)<1\}$ is usually called a "mushy region", carrying a possibly positive energy, that is not high enough to start phase change. Because of this possibly positive energy in the "mushy region" the latent heat is variable. According to the preceding paragraphs, our results will hold for nonnegative solutions $u \in L_{\mathrm{loc}}^{1}\left(\mathbb{R}^{n} \times(0, T)\right)$ which solve (1.1) in the sense of distributions. It follows from lemma 2.4 below that if $u$ is a solution to (1.1), and the initial data $u_{I}$ is a function that does not take values in the open interval $(0,1)$, then the temperature $v=(u-1)_{+}$is a solution in the sense of distributions of the one-phase parabolic combustion problem. This problem can be stated as follows: Find $v=\lim _{\epsilon \rightarrow 0} v^{\epsilon}(x, t)$, where $v^{\epsilon}$ is a solution of

$$
v^{\epsilon}-\Delta v^{\epsilon}(x, t)=\beta_{\epsilon}(v),
$$

and $\beta_{\epsilon}(s)$ appoximates $\delta_{0}(s)$ in $D^{\prime}(\mathbb{R})$.

This problem has been studied recently by many authors (see $[\mathrm{CV}],[\mathrm{Da}]$ ) and the references therein). It was initially believed to be a free boundary 
problem for the heat equation unrelated to the Stefan problem. Solutions of this problem may not be unique, and their support may shrink as time evolves (see $[\mathrm{CV}]$ ).

From the broad literature on the Stefan problem, which was treated extensively, among others, by J. R. Cannon, C. D. Hill, A. Fasano and M. Primicerio, our work is close in its scope to the papers [AtCSa1], [AtCSa2], and [AtCSa3]. In these papers, following the steps of the study of the regularity of minimal surfaces, the authors propose, and pursue a) and b) for viscosity solutions of the two-phase Stefan problem, of the following program:

a) Lipschitz free boundaries are smooth (or are smooth after a certain time. The existence of this positive time, called "waiting time" is typical in parabolic problems.)

b) "Flat" free boundaries (in some Lebesgue differentiability or measure theoretical sense) are Lipschitz.

c) Generalized free boundaries are "flat".

Here we solve c) for the free boundary of the one phase Stefan problem (1.1). Our result will hold for non-negative distributional solutions of (1.1). We show that the free boundary carries a measure $\lambda$ (which accounts for the loss of energy at the phase change). On any set on which an additional condition on the spatial gradient is satisfied (see theorem 2.7 for the precise statement), we show that this measure is carried by an $n$ rectifiable set, that is, that the boundary measure $\lambda$ is $n$ rectifiable (see [Mat], [GiMSou1], or section 2 below, for the precise definitions). Recently, the auhor and C. N. Moore succeeded in removing the condition on the spacial gradient in the statement of theorem 2.7 (see [KMr2]). In remark 2.10 at the end of this paper outline how this is done.

Heuristically, along this rectifiable set the liquid phase advances at finite velocity. The maximum principle shows that once the free boundary reaches (say, at some time $t=t_{0}$ ), a set where the initial datum is a locally integrable function $u_{I}(x)=1$ a. e., that is surrounded by a set where the initial datum is smaller than 1, it advances at infinite velocity - the diffusion is caloric, and the advance is instantaneous. It follows easily from Besicovitch's covering theorem and our framing of the problem in section 2 that the free boundary measure $\lambda$ does not charge such "horizontal" parts of the free boundary. Also $\lambda$ will not charge the set of so-called "focussing points": points $(x, t)$ of the free boundary where a part of the "mushy" region is finally taken over by a surrounding caloric region (see part ii) of theorem 2.7). In view of the geometry of the modulus of continuity of $(u-1)_{+}$, see [Di], and the shape of the parabolic approach "cones", see [K1], such points are typical 
singularities of the free boundary.

It also follows from lemma 2.4 below that $\lambda$ does not charge the interior of vertical segments contained in the free boundary. In particular, at "corner points" the free boundary cannot advance until the caloric region has expanded in such a way that a measure theorethical normal can be defined. Such "waiting times" are another typical singularity encountered in parabolic free boundary problems.

We also want to stress that we do not use a different scale for the time and the space coordinates. While often in parabolic potential theory a modified Hausdorff measure is used, in which the time dimension "counts" double with respect to the dimension in the space variables, we will count the space and time dimensions equally. In fact we will use a variant of "spherical" Hausdorff measure, replacing Euclidean balls $B_{r}^{n+1}\left(x_{0}, t_{0}\right)=\{(x, t): \mid(x, t)-$ $\left.\left(x_{0}, t_{0}\right) \mid<r\right\}$ with cylinders $C_{r}\left(x_{0}, t_{0}\right)=\left\{(x, t):\left|x-x_{0}\right|<r,\left|t-t_{0}\right|<r\right\}$. The use of these cylinders makes computations simpler. Recently, G. Weiss (see $[\mathrm{Ws}]$ ) proved a structure result for the free boundary of the one-phase combustion problem. He studies the limits of sequences of scalings $u_{k}(x, t)=$ $u\left(k x, k^{2} t\right)$ of a solution $u(x, t)$ of the problem (1.2). We feel that both the divergence structure of equation (1.1) (see (2.2)) and the natural bound for the boundary measure (see lemma 2.2 below) make it desirable to work within an euclidean geometry.

Our approach is very different from the one in [AtCSa1], [AtCSa2], and [AtCSa3]: Because our solution is understood in the distribution sense in $\mathbb{R}^{n} \times(0, T)$, the regularity information we obtained holds across the free boundary. In [AtCSa1], [AtCSa2], and [AtCSa3], solutions are viscosity solutions and the free boundary condition is understood in an asymptotic sense. In their setting regularity information for the solutions near the free boundary and regularity of the free boundary are sought for simultaneously.

Our setting includes in a natural way the presence of a "mushy" region (the set where the solution is between 0 and 1 ) where the energy is positive, but not enough to start the phase change. This highlights the hyperbolic aspect of the problem, since in open sets within this region the trivial conservation law $u_{t}=0$ is satisfied.

Our method exploits heavily that the problem is a one-phase one: First, we rely heavily on apriori regularity, and the main steps to obtain it (in particular, the crucial result $L_{\text {loc }}^{1} \Rightarrow L_{\text {loc }}^{2}$, see $[\mathrm{K}]$, require nonnegative solutions. Second and more deeply, the fact that the interphase can only advance is crucial in theorem 2.7, and in the lemmas leading to it (by allowing to define the function $t(x)$ in (2.1)). As a consequence the "boundary" measure is nonnegative, whence its lower $n$ density is nonnegative (see section 2 for the 
definition of $n$ density). This is one of the requirements of Preiss' theorem, which is the main tool in our work. The author and C. N. Moore have since beeen able to adapt the tools and ideas in this paper to the two-phase Stefan problem with a "mushy zone" (that is, allowing for signed solutions), under the assumption that the solution $u \in L_{\text {loc }}^{2}$ (see [KMr1] and [KMr2]). Further developing the ideas in the present paper, the author and D. Danielli were able to show that at most points of the free boundary the classical jump condition is satisfied in a natural (density) sense (see $[\mathrm{DaK}]$ ).

We would like to note that the problem under discussion is related to singular integrals on sets of low regularity, and to oscillatory integrals. This can be seen by integrating the expression (2.2), against the fundamental solution of the heat equation, and a limiting process which in turn uses the regularity information for solutions to equation (1.1) described above. We will not use this approach in this paper.

The method of proof of our result is simple. Basically it reduces to careful handling of the boundary measure $\lambda$. We obtain two disintegrations of this measure in terms of the solution $u$ of (1.1), show that it is supported on the free boundary, and then study its density. Since the boundary measure $\lambda$ needs not satisfy any homogeneous lower bound on balls (i. e. $\lambda$ is not Ahlfors regular), we use the powerful result of D. Preiss ([Pre], see also [Mat]) on rectifiability of measures. As a consequence, we will obtain that but a null set for the measure $\lambda$, (rather than a null set for $n$ dimensional Hausdorff measure) the free boundary is the union of $n$ dimensional Lipschitz images.

Partial results have been announced in [K2]. The author is gratefully indebted to Berndt Kirchheim for helpful suggestions.

\section{Rectifiability of the free boundary measure.}

In this section we will prove our main result, theorem 2.7. After some definitions we will identify the measure supported on the free boundary (lemmas 2.1 and 2.2). The rectifiability of this measure $\lambda$ will follow from Preiss' powerful result (theorem 2.6 below). We will first need to prove some properties of the measure $\lambda$ that we will need in the proof of theorem 2.7. In this theorem we show that $\lambda$ satisfies the hypotheses of Preiss' theorem on rectifiability of measures.

We begin with some general notations and definitions.

Throughout this paper we will write $B^{n+1}\left(\left(x_{0}, t_{0}\right), r\right)$ for the $n+1$ dimensional Euclidean ball centered at $\left(x_{0}, t_{0}\right)$ with radius $r, B^{n}\left(x_{0}, r\right)$ or $B_{r}\left(x_{0}\right)$ for the $n$ dimensional Euclidean ball centered at $x_{0}$ with radius $r$, and $C_{r}\left(x_{0}, t_{0}\right)$ for the cylinder $B_{r}\left(x_{0}\right) \times\left(t_{0}-r, t_{0}+r\right)$. $\mathcal{L}^{n}$ will stand for 
$n$ dimensional Lebesgue measure and $\mathcal{H}^{n}$ for $n$ dimensional Hausdorff measure. For $0 \leq m<\infty$ we define the upper and lower $m$ densities of a Radon measure $\mu$ at a point $a \in \mathbb{R}^{n}$ as

$$
\begin{aligned}
& \theta^{* m}(\mu, a)=\lim \sup _{r \rightarrow 0}(2 r)^{-m} \mu\left(B^{n}(a, r)\right), \\
& \theta_{* m}(\mu, a)=\liminf \inf _{r \rightarrow 0}(2 r)^{-m} \mu\left(B^{n}(a, r)\right) .
\end{aligned}
$$

If they agree, their common value

$$
\theta^{m}(\mu, a)=\theta^{* m}(\mu, a)=\theta_{* m}(\mu, a)
$$

will be called the $m$ density of $\mu$ at $a$. A set $E \subset \mathbb{R}^{n}$ is called $m$ rectifiable if there exist Lipschitz maps $f_{i}: \mathbb{R}^{m} \rightarrow \mathbb{R}^{n}, i=1,2, \ldots$, such that

$$
\mathcal{H}^{m}\left(E \backslash \cup_{i=1}^{\infty} f_{i}\left(\mathbb{R}^{m}\right)\right)=0 .
$$

A set $F \subset \mathbb{R}^{n}$ is called purely $m$ unrectifiable if $\mathcal{H}^{m}(E \cap F)=0$ for every $m$ rectifiable set $E$. A Radon measure $\mu$ on $\mathbb{R}^{n}$ is $m$ rectifiable if $\mu$ is absolutely continuous with respect to $\mathcal{H}^{m}$ and there exists an $m$ rectifiable Borel set $E$ such that $\mu\left(\mathbb{R}^{n} \backslash E\right)=0$. If a set $E$ is an $m$ rectifiable subset of $\mathbb{R}^{n}$, by a result of Federer $([\mathrm{F}]$, Chapter 3$) \mathcal{H}^{m}\left(E \backslash \cup_{i=1}^{\infty} M_{i}\right)=0$, where $M_{i}$ are $m$ dimensional immersed submanifolds of $\mathbb{R}^{n}$.

We define the function

$$
t(x):=\inf \{t>0: u(x, t)>1\}
$$

where $u$ is the solution to (1.1). This function may be zero (for example on sets where the initial trace is a. e. $>1$ ). We will by convention write $t(x)=T$ when $t(x) \notin(0, T)$ (this may happen on sets where the initial trace is $\mathcal{L}^{n}$ a. e. smaller than 1 which are never reached by the diffusion). Here we are using that our solutions are non-negative (that is, that we are working on the one phase case), whence for $t>t(x),(u-1)_{+}(x, t)>0$. Thus we have that the free boundary $\partial\left\{(x, t):(u-1)_{+}(x, t)>0\right\}$ is the union $\mathcal{F}$ of the graph $F=\left\{(x, t) \in \mathbb{R}^{n} \times[0, T]: t=t(x)\right\}$ of the function $t(x)$ with segments that are parallel to the $t$ axis placed at the values of $x$ at which $t(x)$ has a jump.

2.1. Lemma. The function $t(x)$ is upper semicontinuous, whence a Borel function, and therefore measurable.

Proof. The function $(u-1)_{+}$enjoys a uniform modulus of continuity on each compact subset of $\mathbb{R}^{n} \times(0, T)$ (see [Di], [K]). If $x_{0} \in\{t(x)<a\}$, let 
$t_{0} \in\left(t\left(x_{0}\right), a\right)$. There exists $0<\rho<a-t\left(x_{0}\right)$ such that $u(x, t)>1$ for $(x, t) \in B_{\rho}^{n}\left(x_{0}\right) \times\left(t_{0}-\rho, t_{0}+\rho\right)$. In particular, $t(x)>a$ for $x \in B_{\rho}^{n}\left(x_{0}\right)$.

Recall that $(u-1)_{+}$is a (distributional) subsolution to the heat equation (see $[\mathrm{K}])$, that is,

$$
\Delta(u-1)_{+}-\frac{\partial}{\partial t}(u-1)_{+} \geq 0 \quad \text { in } D^{\prime}\left(\mathbb{R}^{n} \times(0, T)\right) .
$$

This naturally defines a (non-negative) Radon measure

$$
\lambda=\Delta(u-1)_{+}-\frac{\partial}{\partial t}(u-1)_{+}=u_{t}-\frac{\partial}{\partial t}(u-1)_{+} .
$$

We next study the measure $\lambda$.

2.2. Lemma. The following holds for $\lambda$ :

i) $\lambda$ is a non-negative Radon measure.

ii) $\operatorname{Supp}(\lambda) \subset \partial\left\{(u-1)_{+}>0\right\}$.

iii) $\left.\lambda\left(B_{r}^{n}\left(x_{0}\right) \times\left(t_{0}-r, t_{0}+r\right)\right)\right) \leq \mathcal{L}^{n}\left(B_{r}^{n}\right)=c_{n} r^{n}$, whence $\lambda$ is a Carleson measure (see $[\mathrm{St}])$. This estimate also shows that $\lambda$ defines a continuous linear functional on $B V\left(\mathbb{R}^{n} \times(0, T)\right.$ ) (see [Z], theorem 5.12.4). Moreover,

$$
\theta^{* n}\left(\lambda,\left(x_{0}, t_{0}\right)\right)=\lim \sup _{r \rightarrow 0} \frac{1}{2^{n} r^{n}}\left(\lambda\left(C_{r}\left(x_{0}, t_{0}\right)\right) \leq \frac{c_{n}}{2^{n}}\left(1-u_{I}\left(x_{0}\right)\right)_{+}<\infty\right.
$$

for $\mathcal{L}^{n}$ a. e. $x_{0} \in \mathbb{R}^{n}$, where $c_{n}=\mathcal{L}^{n}\left(B_{1}^{n}(0)\right)$.

iv) $\lambda=\operatorname{div}_{(x, t)}(V)$, with

$$
V(x, t)=\left(\nabla(u-1)_{+},-(u-1)_{+}\right) \in H_{\mathrm{loc}}^{1}\left(\mathbb{R}^{n} \times(0, T)\right) .
$$

In particular, $\lambda \in H_{0}^{1}\left(\mathbb{R}^{n} \times(0, T)\right)^{*}$, and for $f \in H_{0}^{1}\left(\mathbb{R}^{n} \times(0, T)\right)$,

$$
(\lambda, f)=-\left(V, \nabla_{(x, t)} f\right) .
$$

\section{Proof.}

i) Is obvious and the motivation for the defintion of $\lambda$.

ii) Since $(u-1)_{+}$solves the heat equation in the open set

$$
\left\{(x, t):(u-1)_{+}>0\right\},
$$


we have that

$$
\lambda\left(\left\{(x, t):(u-1)_{+}>0\right\}\right)=0 .
$$

If $B_{r}\left(x_{0}\right) \times\left(t_{0}-r, t_{0}+r\right)$ is contained in the interior of $\left\{(u-1)_{+}=0\right\}$, then for $\mathcal{L}^{n}$ a. e. $x \in B_{r}\left(x_{0}\right)$, the function $u(x, t)$ is constant in $t$, and actually a. e. equal to the initial data $\left.u_{I}(x)\right)$ for $0<t<t_{0}$. This follows from local uniqueness (see $[\mathrm{K}]$ ) and the fact that $u(x, t)=u_{I}(x)$ solves the equation (1.1) in the sense of distributions in $\left.B_{r}\left(x_{0}\right) \times\left(0, t_{0}\right)\right)$. Using the right hand side expression of $\lambda$ in (2.2), we see that $\lambda\left(B_{r}\left(x_{0}\right) \times\left(0, t_{0}\right)\right)=0$.

iii) Using the expression of $\lambda$ in the right hand side of (2.1), and recalling that $u(x, t)$ is non decreasing in $t$ for a. e. $x$ in measurable subsets of $\{(x, t)$ : $u(x, t)<1\}$, we obtain that

$$
\begin{gathered}
\lambda\left(B_{r}\left(x_{0}\right) \times\left(0, t_{0}\right)\right) \leq \int_{B_{r}\left(x_{0}\right)} \frac{\partial}{\partial t}\left(u-(u-1)_{+}\right) \leq \\
\leq \int_{B_{r}\left(x_{0}\right)}\left(1-u_{I}(x)\right)_{+} d x \leq \mathcal{L}^{n}\left(B_{r}\left(x_{0}\right)\right) .
\end{gathered}
$$

iv) Is obvious since we showed in $[\mathrm{AnK}]$ that $(u-1)_{+} \in H_{\mathrm{loc}}^{1}\left(\mathbb{R}^{n} \times(0, T)\right)$.

2.3 Remarks. As a consequence of i) and iii) above, $\lambda \ll \mathcal{H}^{n}$, whence $\lambda$ does not charge any sets of Hausdorff dimension lower than $n$. Recalling that $\frac{\partial}{\partial t}(u-1)_{+} \in L_{l o c}^{2}\left(\mathbb{R}^{n} \times(0, T)\right)$, and $u_{t}=\lambda+\frac{\partial}{\partial t}(u-1)_{+}$, we see that the absolutely continuous part of $u_{t}$ is supported in $\{u>1\}$, and the singular part on the free boundary $\mathcal{F}=\partial\left\{(u-1)_{+}>0\right\}$.

The fact that $\lambda$ defines a linear continuous functional on $B V\left(\mathbb{R}^{n}\right)$, says that, heuristically, test functions in $B V$ should suffice to retrieve all the information $\lambda$ carries. This motivated the author to seek the structure theorem 2.7 below.

The computation in the proof of iii) above shows the convenience of using cylinders $C_{r}$ instead of balls $B_{r}^{n+1}$ to exploit best the geometry of $\lambda$. The $\mathcal{L}^{n+1}$ measures of the cylinders are proportional to the $\mathcal{L}^{n+1}$ measures of the balls. Therefore throughout this work we will use the variant of spherical Hausdorff measure obtained employing such cylinders in the coverings instead of balls. We will not keep track of the proportionality constants. We stress again that we do not modify the time scale in these cylinders.

Next we will disintegrate $\lambda$ in a convenient way for its further study.

2.4. Lemma. The measure $\lambda$ has the decomposition $\lambda=\left(\left(1-u_{I}(x)\right)_{+} \delta_{t=t(x)}\right) d x+\left(V_{0}^{t(x)-}\left(u_{t}-\frac{\partial}{\partial t}(u-1)_{+}\right)\right)(x, s) d x=\lambda_{1}+\lambda_{2}$, 
where $V_{0}^{t(x)-}\left(u_{t}-\frac{\partial}{\partial t}(u-1)_{+}\right)$stands for the total variation over the interval $[0, t(x)) \cap[0, s]$ of the measure defined for a. e. $x$ by $u_{t}-\frac{\partial}{\partial t}(u-1)_{+}$. Moreover,

$$
\lambda_{1}\left(C_{r}\right)=\int_{\pi\left(C_{r} \cap F\right)}\left(1-u_{I}(x)\right)_{+} d x \leq c_{n} r^{n},
$$

for any cylinder $C_{r}\left(x, t_{0}\right)=B^{n}\left(x_{0}, r\right) \times\left(t_{0}-r, t_{0}+r\right)$. Here $\pi: \mathbb{R}^{n+1} \rightarrow \mathbb{R}^{n}$ stands for the projection on $\mathbb{R}^{n}, \pi(x, t)=x$.

Proof. The decomposition above follows from the fact that $u(x, t)$ is nondecreasing in $\{(x, t): u(x, t)<1\}$ but constant in the interior of $\left\{(x, t):(u(x, t)-1)_{+}=0\right\}$.

From (2.3), $\lambda_{1}$ is a "weighted" Young measure (see e. g. [GiMSou1] for the definition of Young measure). It also follows from lemma 2.4 and (2.3) that if $u_{I}$ does not take values in the interval $(0,1)$ and $\lambda_{2}=0$ then $v=(u-1)_{+}$ is a solution to the one phase combustion problem, see $[\mathrm{CV}]$.

2.5. Lemma. The measure $\bar{\lambda}_{2}(x)=\left(V_{0}^{t(x)-}\left(u_{t}-\frac{\partial}{\partial t}(u-1)_{+}\right)\right)(x, t(x)) d x$ (the marginal of $\lambda_{2}$ ) is carried by a $\mathcal{L}^{n}$ null set.

Proof. Let $F$ denote the graph of the function $t(x)$, and $F_{1}=\mathcal{F} \backslash F$ the set of segments of the free boundary parallel to the $t$ axis. Then for any ball $B\left(x_{0}, r\right)$,

$$
\bar{\lambda}_{2}\left(B\left(x_{0}, r\right)\right)=\int_{B\left(x_{0}, r\right)} \chi_{\pi\left(F_{1}\right)}(x) V_{0}^{t(x)-}\left(u_{t}\right)(x, s) d x .
$$

If for some $x_{0} \in \mathbb{R}^{n}, \theta^{* n}\left(\bar{\lambda}_{2}, x_{0}\right)>0$, then $\pi\left(F_{1}\right)$ has to have positive $\mathcal{L}^{n}$ density at $x_{0}$.

That is, $x_{0} \in S$, where

$$
S=\{x: \text { ap limsup } t(x)-\operatorname{ap} \lim \inf t(x)>0\},
$$

and $S \cap F_{1}$ has positive $\mathcal{L}^{n}$ density at $x_{0}$. But this cannot happen because $t(x)$ is measurable and therefore approximately continuous at a. e. $x$.

Next let us recall Preiss' theorem on rectifiability of measures $([\mathrm{P}]$, also Thm. 17.8 in $[\mathrm{M}])$.

2.6. Theorem (Preiss [Pre]). Let $\mu$ be a Radon measure on $\mathbb{R}^{n}$ such that the density $\theta^{m}(\mu,(x, t))$ exists and is positive and finite for $\mu$ almost all $(x, t) \in \mathbb{R}^{n}$. Then $\mu$ is $m$ rectifiable. 
2.7. Theorem. Let $E \subset \mathbb{R}^{n} \times(0, T)$ such that for every $\left(x_{0}, t_{0}\right) \in E \cap F$, $\liminf _{r \rightarrow 0}\left\{\left|\nabla(u-1)_{+}(x, t)\right|,(x, t) \in C_{r}\left(x_{0}, t\left(x_{0}\right)\right)\right\} \geq b$ for some $0<b<\infty$. Then the boundary measure $\lambda$ restricted to $E$ is $n$ rectifiable.

Proof. By lemma 2.5 and Besicovitch's covering lemma,

$$
\lambda_{2}\left(\left\{(x, t): \theta^{* n}\left(\lambda_{2}\right)(x, t)=0\right\}\right)=0,
$$

so we only need to worry about the rectifiability of $\lambda_{1}$.

All we need to check is that $\theta^{n}\left(\lambda_{1},(x, t)\right)$ exists and is positive and finite for $\lambda_{1}$ almost all $(x, t) \in \mathbb{R}^{n} \times(0, T)$, i. e. that the set of points where either the upper and lower densities of $\lambda_{1}$ do not coincide, or the upper density is 0 , has $\lambda_{1}$ measure 0 . Since $\lambda_{1}$ does not charge the interior of any segment parallel to the $t$ axis contained in $\mathcal{F}$, we only need to worry about the points of the graph of $t(x)$. Also it suffices to show that the theorem holds in any cylinder $C=B_{R}(0) \times(a, b) \subset \mathbb{R}^{n} \times(0, T)$, since we can exhaust the whole strip with an increasing collection of such cylinders. We will only consider points $\left(x_{0}, t\left(x_{0}\right)\right)$ such that $x_{0}$ is a Lebesgue point of $\left(1-u_{I}(x)\right)_{+}$, and the precise representative of $\left(1-u_{I}(x)\right)_{+}>0$ (we will always work with the precise representatives of functions in what follows) since by Besicovitch's covering lemma, or simply by the desintegration of $\lambda_{1}$ in the proof of iv) in lemma 2.2, $\lambda_{1}$ will not charge the set

$$
\left\{(x, t): x \text { is not a Lebesgue point of }\left(1-u_{I}\right)_{+}\right\} .
$$

A similar reasoning shows that $\lambda_{1}$ does not charge the set

$$
\left\{(x, t) \in \mathbb{R}^{n} \times(0, T):\left(1-u_{I}(x)\right)_{+}=0\right\} .
$$

Let us divide the proof in steps:

i) Let $B_{1}=\left\{(x, t(x)) \in C \cap F: \theta_{* n}\left(\lambda_{1},(x, t(x))<\theta^{* n}(x, t(x))\right\}\right.$. By a standard argument, $B_{1}$ will be written as a countable union of sets

$$
B_{p, q}=\left\{(x, t(x)) \in B_{1}: \theta_{* n}\left(\lambda_{1},(x, t(x))<p<q<\theta^{* n}(x, t(x))\right\},\right.
$$

where $p, q$ are rational numbers. In $B_{p, q}, q<\theta^{* n}(x, t(x)) \leq c_{n} 2^{n}$ holds, whence on this set, $\mathcal{H}^{n}$ and $\lambda_{1}$ are mutually absolutely continuous, in particular, $\mathcal{H}^{n}\left(B_{p, q}\right)<\infty$. Then, by Federer's structure theorem, $B_{p, q}=M \cup N$, where $M$ is $n$ rectifiable and $N$ has integral-geometric (or Favard) measure 0. At $\mathcal{H}^{n}$ almost every point, $M$ has an approximate tangent plane. It is easy to verify that at every point $\left(x_{0}, t\left(x_{0}\right)\right)$ of $F$ admitting a measure theoretic 
tangent plane, $x_{0}$ being a Lebesgue point of $\left(1-u_{I}\right)_{+}$, the density of $\theta^{n} \lambda_{1}$ exists, a contradiction. Therefore $\mathcal{H}^{n}(M)=0$, and the same holds for $\lambda_{1}$.

Next let us show that $\lambda_{1}(N)=0$. If $\mathcal{L}^{n}(\pi(N))=0$ we have nothing to prove. Assume $\mathcal{L}^{n}(\pi(N))>0$. If we rotate the $t$ axis onto the line $t_{\omega}$ by a sufficientntly small angle $\omega$, since $N \subset F$, its projection on the hyperplane $P_{\omega}$ nomal to the direction $t_{\omega}$ has positive $\mathcal{H}^{n}$ measure, and we can recover the $\mathcal{H}^{n}$ measure of the projection of $N$ on this new hyperplane by using change of variables. The Jacobian of the transformation of $\pi(N)$ into the new hyperplane is a continuous function of $\omega$ with value 1 for $\omega=0$. But then $N$ has to have stricty positive integral-geometric measure. We conclude that $\mathcal{L}^{n}(N)=0$.

This shows that $\lambda_{1}\left(B_{1}\right)=0$.

ii) Let $B_{2}=\left\{(x, t(x)): \theta^{* n}\left(\lambda_{1},(x, t(x))\right)=0\right\}$. Of course we may assume that

$$
\lim _{r \rightarrow 0} \frac{1}{\mathcal{L}^{n}\left(B_{r}\left(x_{0}\right)\right)} \int_{B_{r}\left(x_{0}\right)}\left(1-u_{I}(x)\right)_{+} d x=\left(1-u_{I}(x)\right)_{+}>0,
$$

and that $x$ is a point of approximate continuity of $t(x)$. Then, for $\theta^{* n}\left(\lambda_{1},\left(x_{0}, t\left(x_{0}\right)\right)\right)=0$ to hold,

$$
\lim _{r \rightarrow 0} \frac{\mathcal{L}^{n}\left(\pi\left(C_{r} \cap F\right)\right)}{\mathcal{L}^{n}\left(B_{r}\right)}=0
$$

has to hold.

2.8. Claim. We claim that if

$$
\lim _{r \rightarrow 0} \frac{\mathcal{L}^{n}\left(\pi\left(C_{r} \cap F\right)\right)}{\mathcal{L}^{n}\left(B_{r}\right)}=0,
$$

then

$$
\lim _{r \rightarrow 0} \frac{\mathcal{L}^{n+1}\left(C_{r} \cap\{(x, t): t>t(x)\}\right)}{\mathcal{L}^{n+1}\left(C_{r}\right)}=1 .
$$

We pospone the proof of claim 2.8 to the of the paper end return to the proof of theorem 2.7:

Let $\left.S_{1}=\{(x, t): t>t(x)\} \subset \mathbb{R}^{n} \times[0, T]\right\}$, and let

$$
M=\left\{x \in \mathbb{R}^{n}: D^{n+1}\left(S_{1},(x, t(x))=1\right\},\right.
$$

where $D^{n+1}$ stands for $\mathcal{L}^{n+1}$ density. We will show that $\mathcal{L}^{n}(M)=0$. 
Indeed, fix $\alpha$ small (to be chosen later), and let

$$
M_{k}=\left\{x: \frac{\mathcal{L}^{n+1}\left(B(x, r) \times(t(x)-r, t(x)+r) \backslash S_{1}\right)}{\mathcal{L}^{n+1}(B(x, r) \times(t(x)-r, t(x)+r))}<\alpha, \forall r \in\left(0, \frac{1}{k}\right)\right\} .
$$

If $\mathcal{L}^{n}(M)>0$, then there exists $k_{0}$ such that $\mathcal{L}^{n}\left(M_{k_{0}}\right)>0$. Choose a disk in $\mathbb{R}^{n}, B=B(y, r), r<\frac{1}{2 k_{0}}$, such that $B \cap M_{k_{0}} \neq \phi$. There exists $x_{0} \in B \cap M_{k_{0}}$ with

$$
t\left(x_{0}\right)-\frac{r}{8}<\inf t\left(B \cap M_{k_{0}}\right) .
$$

Then,

$$
\left(\{y\} \times\left(0, t\left(x_{0}\right)-\frac{r}{8}\right)\right) \cap S_{1}=\phi,
$$

for all $y \in B \cap M_{k_{0}}$. We conclude that

$$
\mathcal{L}^{n}\left(M_{k_{0}} \cap B_{r}\right) \frac{15}{8} r \leq \mathcal{L}^{n+1}\left(C_{2 r} \backslash S_{1}\right)<\alpha \mathcal{L}^{n+1}\left(C_{2 r}\right) .
$$

After some manipulation this yields

$$
\mathcal{L}^{n}\left(B_{r} \cap M_{k_{0}}\right) \leq\left(\alpha c 2^{n+1} \frac{8}{15}\right) \mathcal{L}^{n}\left(B_{r}\right) .
$$

Choosing now $\alpha<\left(c 2^{n+1} \frac{8}{15}\right)^{-1}$, this implies that $\mathcal{L}^{n}\left(M_{k_{0}}\right)=0$.

A similar argument shows that

$$
\mathcal{L}^{n}\left(\left\{x \in \mathbb{R}^{n}: D\left(S_{2},(x, t(x))=1\right\}\right)=0,\right.
$$

where $S_{2}=\{(x, t): t<t(x)\}$.

Note that in the density topology, points in $B_{2}$ are actually interior points of one of the phases.

Proof of Claim 2.8. We will use De Giorgi's lemma, which we state next (see [LSolU], p. 89):

2.9. Lemma (De Giorgi). Let $B_{R} \subset \mathbb{R}^{m}$ be a ball, $v \in H^{1}\left(B_{R}\right)$ and let $k, l$ be real numbers such that $l>k$, and write $A_{k, R}=\left(\left\{y \in B_{R}, v(y)>k\right\}\right.$ for any $k \in \mathbb{R}$. Then

$$
(l-k) \mathcal{L}^{m}\left(A_{l, R}\right) \mathcal{L}^{m}\left(B_{R} \backslash A_{k, R}\right) \leq D R^{m+1} \int_{A_{k, R} \backslash A_{l, R}}|\nabla v| d y,
$$

where $D$ is a constant depending only on the dimension $m$. 
First note that for $x_{0} \in \mathbb{R}^{n}, t_{0} \in(0, T)$ and $r>0$ such that $0<t_{0}-r$, and $t_{0}+r<T$,

$$
\mathcal{L}^{n}\left(\left\{\left(x, t_{0}+r\right), x \in B_{r}\left(x_{0}\right):(u-1)_{+}\left(x, t_{0}+r\right)>0\right\}\right)=
$$

$\mathcal{L}^{n}\left(\left\{\left(x, t_{0}-r\right), x \in B_{r}\left(x_{0}\right):(u-1)_{+}\left(x, t_{0}-r\right)>0\right\}\right)+\mathcal{L}^{n}\left(\pi\left(C_{r} \cap \operatorname{Graph}(t)\right)\right)$.

Also

$$
\mathcal{L}^{n}\left(\left\{(x, s), x \in B_{r}\left(x_{0}\right):(u-1)_{+}(x, s)>0\right\}\right)
$$

is increasing in $s$. Then by (2.4) there exists $1>a>0$ such that for $\epsilon>0$, $a+\epsilon<1, r<r_{0}$ small enough, and $t_{0}-r<t<t_{0}+r$,

$$
(a+\epsilon) \mathcal{L}^{n}\left(B_{r}\right) \geq \mathcal{L}^{n}\left(\left\{(x, t), x \in B_{r}\left(x_{0}\right):(u-1)_{+}(x, t)=0\right\}\right) \geq a \mathcal{L}^{n}\left(B_{r}\right) .
$$

For a. e. $t \in\left(t_{0}-r, t_{0}+r\right)$ we apply De Giorgi's lemma to $v(x)=(u-$ $1)_{+}(x, t)$, with $k=h, l=h / 2$ to obtain

$$
c_{n} r^{2 n} a(1-a-\epsilon) \leq D r^{n+1} \frac{2}{h} \int_{C_{r} \cap\left\{h / 2<(u-1)_{+}<h\right\}}\left|\nabla(u-1)_{+}\right|(\cdot, t) d x,
$$

where $c_{n}$ denotes a positive constant depending only on $n$ that may change from line to line. Integration over $\left(t_{0}-r, t_{0}+r\right)$ gives

$$
\begin{gathered}
c_{n} r^{2 n+1} a(1-a-\epsilon) \leq \\
D r^{n+1} \frac{2}{h} \int_{C_{r} \cap\left\{h / 2<(u-1)_{+}<h\right\}}\left|\nabla(u-1)_{+}\right|(x, t) d x d t .
\end{gathered}
$$

Then

$$
\begin{gathered}
c_{n} a(1-a-\epsilon) \leq \\
\lim _{r \rightarrow 0} \lim _{h \rightarrow 0} r^{-n} \frac{2}{h} \int_{C_{r} \cap\left\{h / 2<(u-1)_{+}<h\right\}}\left|\nabla(u-1)_{+}\right|(x, t) d x d t .
\end{gathered}
$$

For $h>0$ set

$$
\phi_{h}(x)= \begin{cases}1 & \text { if } x>h \\ \frac{2}{h} x-\frac{h}{2} & \frac{h}{2} \leq x<h \\ 0 & \text { if } x<\frac{h}{2}\end{cases}
$$

Let $0 \leq \eta \in \mathcal{D}\left(\mathbb{R}^{n} \times(O, T)\right)$. Then $\eta \phi_{h}\left((u-1)_{+}\right)$is supported in $\left\{(u-1)_{+} \geq \frac{h}{2}\right\}$; in particular, $\left(\lambda, \eta \phi_{h}\left((u-1)_{+}\right)=0\right.$ since $\lambda=\Delta(u-1)_{+}-$ $\frac{\partial}{\partial t}(u-1)_{+}=\frac{\partial}{\partial t}\left(u-(u-1)_{+}\right)$is supported on $\left\{(u-1)_{+}=0\right\}$. Using $\eta \phi_{h}((u-$ $\left.1)_{+}\right)$as test functions one can show (see $\left.[\mathrm{KMr} 1]\right)$ that

$$
\lim _{h \rightarrow 0} \frac{2}{h} \int_{C_{r} \cap\left\{h / 2<(u-1)_{+}<h\right\}}\left|\nabla(u-1)_{+}\right|^{2}(x, t) d x d t=\lambda\left(C_{r}\right) .
$$


Then the right hand side in (2.6) is bounded by

$$
\begin{gathered}
\frac{1}{b} \lim _{r \rightarrow 0} \lim _{h \rightarrow 0} r^{-n} \frac{2}{h} \int_{C_{r} \cap\left\{h / 2<(u-1)_{+}<h\right\}}\left|\nabla(u-1)_{+}\right|^{2}(x, t) d x d t= \\
\frac{1}{b} \lim _{r \rightarrow 0} \frac{\lambda\left(C_{r}\right)}{r^{n}}=0 .
\end{gathered}
$$

But then $a=0$, which proves the claim.

2.10. Remark (see $[\mathbf{K M r 2}]$ ). To remove the assumption on the existence of a positive lower bound for $\left|\nabla(u-1)_{+}\right|$in a neighborhood of $\left(x_{0}, t_{0}\right)$, apply Cauchy-Schwarz' inequality to the right hand side of (2.6). Notice that

$$
\begin{gathered}
\mathcal{L}^{n}\left(\pi\left(F \cap C_{r}\right)\right)=\lim _{\delta \rightarrow 0} \int_{B_{r}\left(x_{0}\right)} \chi_{t^{-1}\left(t_{0}-r, t_{0}+r\right)}(x) \chi_{\left\{(u-1)_{+}=\delta\right\}}(x, t(x)) d x= \\
\lim _{\delta \rightarrow 0} \int_{t_{0}-r}^{t_{0}+r} \int_{\left\{(u-1)_{+}(\cdot, t)=\delta\right\}} \frac{\chi_{B_{r} \times\{t\}}(x)}{\left|\nabla(u-1)_{+}(\cdot, t)\right|} d \mathcal{H}^{n-1} d t,
\end{gathered}
$$

where we have used the co-area formula, lemma 2.5, the fact the level sets within the diffusive region are smooth, and the boundary point lemma (see e.g. [PoWn], p.170). In addition,

$$
\begin{gathered}
\liminf _{h \rightarrow 0} \frac{2}{h} \int_{B_{r} \times\{t\}} \chi_{\left\{\frac{h}{2}<(u-1)_{+}(\cdot, t)<h\right\}} d x= \\
\lim \inf _{h \rightarrow 0} \int_{\left\{(u-1)_{+}(\cdot, t)=s_{h}\right\}} \frac{\chi_{B_{r} \times\{t\}}(x)}{\left|\nabla(u-1)_{+}(\cdot, t)\right|} d \mathcal{H}^{n-1} .
\end{gathered}
$$

Integrating in $t$ over $\left(t_{0}-r, t_{0}+r\right)$, use of Fatou's lemma and the fact that

$$
\lim _{r \rightarrow 0} \frac{\mathcal{L}^{n}\left(\pi\left(F \cap C_{r}\right)\right)}{r^{n}}=0
$$

complete the proof (we actually only need the boundedness of the last quantity). The other factor is $\theta^{n}\left(x_{0}, t\left(x_{0}\right)\right)=0$.

\section{References.}

[AtCSa1] I. Athanasopoulos, L. Caffarelli, S. Salsa, Regularity of the free boundary in parabolic phase transition problems, Acta Math. 176 (1996), no. 2, 245-282. 
[AtCSa1] I. Athanasopoulos, L. Caffarelli, S. Salsa, Caloric functions in Lipschitz domains and the regularity of solutions of phase transition problems, Ann. of Math. (2) 143 (1996), no. 3, 413434.

[AtCSa3] I. Athanasopoulos, L. Caffarelli, S. Salsa, Phase transition problems of parabolic type: flat free boundaries are smooth, Comm. Pure Appl. Math. 51 (1998), no. 1, 77-112.

[AnK] D. Andreucci, M. K. Korten, Initial traces of solutions to a one-phase Stefan problem in an infinite strip, Rev. Mat. Iberoamericana, Vol. 9, No. 2 (1993), 315-332.

[CV] L. A. Caffarelli, J. L. Vázquez, A free-boundary problem for the heat equation arising in flame propagation, Trans. Am. Math. Soc. 347, No. 2 (1995), 411-441.

[Da] D. Danielli, A singular perturbation approach to a two phase parabolic free boundary problem arising in combustion theory, to appear in Diff. and Integral Equations.

[DaK $]$ D. Danielli, M. K. Korten, On the pointwise jump condition at the free boundary in the 1-phase Stefan problem, to appear in Comm. in Pure and Appl. Analysis.

[Di] E. DiBenedetto, Continuity of weak solutions to certain singular parabolic equations, Ann. Mat. Pura Appl. (4) 130 (1982), $131-176$.

[EGar] C. L. Evans, R. Gariepy, Measure theory and fine properties of functions, CRC Press, Boca Raton 1992.

[GiMSou1] M. Giaquinta, G. Modica, J. Souček, Cartesian currents in the calculus of variations I, Springer-Verlag Berlin Heidelberg 1998.

[K] M. K. Korten, Non-negative solutions of $u_{t}=\Delta(u-1)_{+}$: Regularity and uniqueness for the Cauchy problem, Nonl. Anal., Th., Meth. and Appl, Vol. 27, No. 5, 589-603 (1996).

[K1] M. K. Korten, A Fatou theorem for the equation $u_{t}=\Delta(u-$ 1) + , Proc. Am. Math. Soc., Vol 128, No. 2, 439-444 (2000). 
[K2] M. K. Korten, On a structure theorem for some free boundary problems for the heat equation, in Free Boundary Problems: theory and applications, I. Athanasopoulos, G. Makrakis, J. F. Rodrigues (eds.), Chapman \& Hall/CRC Research Notes in Mathematics, 155-163 (1999).

[KMr1] M. K. Korten, C. N. Moore, On the Cauchy Problem for the two phase Stefan problem, submitted.

[KMr2] M. K. Korten, C. N. Moore, On the free boundary of the two phase Stefan problem, preprint.

[LSolU] O. A. Ladyženskaja, V. A. Solonnikov, N. N. Ural'ceva, Linear and quasilinear equations of parabolic type, Translations of Mathematical Monographs, Vol. 23, American Mathematical Society, Providence, R.I. 1967.

[Mat] P. Mattila, Geometry of sets and measures in euclidean spaces, Cambridge studies in advanced mathematics 44, Cambridge University Press 1995.

[Pre] D. Preiss, Geometry of measures in $\mathbb{R}^{n}$ : distribution, rectifiability and densities, Ann. of Math. 125 (1987), 537-642.

[ProWn] M. H. Protter, H. F. Weinberger, Maximum Principles in Differential Equations, Springer Verlag Nw York Inc. 1984.

[St] E. Stein, Harmonic Analysis: real-variable methods, orthogonality, and oscillatory integrals, Princeton University Press, Princeton, NJ, 1993.

[Ws] G. Weiss, A singular limit arising in combustion theory: fine properties of the free boundary, Calc. Var. 17, 311-340 (2003).

[Z] W. P. Ziemer, Weakly differentiable functions, Springer-Verlag New York Inc. 1989.

Department of Mathematics

Kansas State University

MANHATtAN, KS 66506-2606

USA

marianne@math.ksu.edu

ReCEIVED ApRIL 2003. 